This is the post print version of the article, which has been published in Comprehensive psychiatry 2018, 84, 26-31.

\title{
Temperament and character profiles are associated with depression outcome in psychiatric secondary care patients with harmful drinking
}

Vesa Paavonen ${ }^{a 1}$, Kaisa Luoto ${ }^{a}$, Antero Lassilac, Esa Leinonen ${ }^{a, b}$, Olli Kampman ${ }^{a, c}$

${ }^{a}$ University of Tampere, Faculty of Medicine and Life Sciences, FI-33014 Tampere, Finland

${ }^{\mathrm{b}}$ Tampere University Hospital, Department of Psychiatry, FI-33014 Tampere, Finland

c Seinäjoki Hospital District, Department of Psychiatry, Huhtalantie 53, 60220 Seinäjoki, Finland

\footnotetext{
${ }^{1}$ Corresponding author: Vesa Paavonen; Address: University of Tampere, Faculty of Medicine and Life Sciences, PO Box 100, FI-33014 Tampere, Finland; Email: vesa.paavonen@outlook.com; Tel: +358407389051; Fax: +358335516164

(C) 2018. This manuscript version is made available under the CC-BY-NC-ND 4.0 license http://creativecommons.org/licenses/by-nc-nd/4.0/
} 


\section{Abstract}

Background: Temperament and character profiles have been associated with depression outcome and alcohol abuse comorbidity in depressed patients. How harmful alcohol use modifies the effects of temperament and character on depression outcome is not well known. Knowledge of these associations could provide a method for enhancing more individualized treatment strategies for these patients.

Methods: We screened 242 depressed patients with at least moderate level of depressive symptoms. The Alcohol Use Disorders Identification Test (AUDIT) was used for identifying patients with marked alcohol use problems (AUP, AUDIT $\geq 11$ ). After 6 weeks of antidepressive treatment 173 patients were assessed using the Montgomery-Åsberg Depression Rating Scale (MADRS), and the Temperament and Character Inventory (TCI-R). Outcome of depression (MADRS scores across three follow-up points at 6 weeks, 6 months and 24 months) was predicted with AUP, gender, and AUP $x$ Gender and AUP $x$ Time interactions together with temperament and character dimension scores in a linear mixed effects model.

Results: Poorer outcome of depression (MADRS scores at 6 weeks, 6 months and 24 months) was predicted by AUP $x$ Time interaction ( $p=0.0002$ ) together with low Reward Dependence ( $p=0.003)$. Gender and all other temperament and character traits were non-significant predictors of the depression outcome in the mixed effects model.

Conclusions: Possibly due to the modifying effect of alcohol use problems, high Reward Dependence was associated with better depression treatment outcome at 6 months. Harm Avoidance and Self-Directedness did not predict depression outcome when alcohol use problems were controlled.

Key words: major depressive disorder; alcohol abuse; Reward Dependence; Self-Directedness; Harm Avoidance; TCl-R 


\section{Introduction ${ }^{2}$}

It is well known that comorbidity of major depressive disorder (MDD) with substance use disorders (SUDs) can lead to impaired response to treatment, more chronic disease courses or recurrence of depressive episodes [1,2]. Because recurrent and chronic depression constitute a major burden of disease [1,2], more efficient treatment strategies and means for earlier identification of high-risk patients are needed.

Assessment of individual temperament and character traits could provide a method for enhancing preventive and more individualized treatment strategies for these patients because certain traits and temperaments may predispose individuals to recurrence of depression, and are associated with different courses of depression and with substance use disorder comorbidity and drinking outcomes [3-6]. Individual personality could have pathoplastic effects on recovery from depression; i.e., differences in temperament or character could explain differences in the course of the illness [7]. How harmful alcohol use modifies the effects of temperament and character on depression outcome is not well known.

The Temperament and Character inventory (TCl-R) is a 240 -item questionnaire that collects information on human personality in the context of temperament and character. According to Cloninger's psychobiological model, on which the TCl-R was based, temperament is divided into four dimensions: Novelty Seeking (NS), Harm Avoidance (HA), Reward Dependence (RD) and Persistence (P). Character is divided into three dimensions: Self-Directedness (SD), Cooperativeness (C) and Self-Transcendence (ST) [8]. These dimensions are thought to reflect combinations of different neurocognitive functions (e.g., memory or reward functions), and the biological basis of this model is supported by many findings $[9,10]$.

According to Cloninger's theory, temperament dimensions generally represent a stable part of personality, and only HA has shown clear state-dependent changes during depression [4,5,8]. However, high HA - and more particularly its sub-scores, anticipatory worry (HA1) and fatigability (HA4) - have also manifested as

\footnotetext{
${ }^{2}$ Abbreviations: MDD = major depressive disorder, SUD = substance use disorder, NS = Novelty Seeking, HA = Harm Avoidance, $\mathrm{RD}=$ Reward Dependence, $\mathrm{P}=$ Persistence, $\mathrm{SD}=$ Self-Directedness, $\mathrm{C}=$ Cooperativeness, $\mathrm{ST}=\mathrm{Self}-$ Transcendence, AUP = alcohol use problems, MADRS = Montgomery-Åsberg Depression Rating Scale, TCI-R = Temperament and Character Inventory
} 
trait-like markers for risk of depression, i.e., index episodes, relapses or recurrent episodes and impaired treatment response $[4,5]$. High RD could be protective against depression in general population, but no associations have been reported with outcome of depression in clinically depressed patients [4,11-15]. Our earlier study of this patient sample suggested that RD is associated with depression treatment outcome in patients with alcohol use problems as change in RD was strongly associated with acute treatment response (0-6 weeks) to depression when alcohol use was taken into account [16]. High NS is a trait indisputably associated with risk of substance use disorders, more severe symptomatology and poorer outcome in SUD patients, and apparently experienced at a higher level in patients with dual diagnosis (concurrent SUD and mental illness) than in depressed patients $[3,6,17-20]$. Low $\mathrm{P}$ and high $\mathrm{HA}$ are associated with more severe alcohol dependence symptomatology [21-23].

Of the character traits, low SD is the trait most clearly predisposing to depression and recurrence of episodes, possibly because individuals with deficiencies in sub-traits such as self-acceptance, responsibility, goal-directedness associated with SD may be more prone to depression due to difficult situations encountered in their daily lives $[24,25]$. There is less evidence to suggest that low $C$ is associated with the development of depression, whereas findings on the associations between ST and depression in different patient samples have been contradictory $[4,15,26,27]$. Low SD is also associated with more severe symptomatology and drinking outcomes in SUD patients $[6,28,29]$. In alcohol dependence character profile with high ST and low SD and C is associated with depression and anxiety [21].

In spite of a large body of knowledge of different associations separately between temperament and character traits and depression or substance use disorders, we found no follow-up studies addressing the associations between depression outcome and temperament, character and alcohol use. We investigated whether temperament and character trait scores (at 6 weeks) together with harmful alcohol use predict outcome of depression in follow-up from 6 weeks to 6 months and to 24 months in a clinically diverse sample of depressed patients. In light of earlier evidence we hypothesize that high HA and low SD and harmful alcohol use together explain poorer depression treatment outcome (measured as MADRS scores) 
$[4,5,25]$. As harmful alcohol use had a modifying effect on both temperament and character dimensions during acute illness, we hypothesize that RD together with alcohol use is also associated with outcome of depression in the long-term follow-up (from 6 weeks to 6 and 24 months) [16]. High NS has been associated with more severe SUDs, and therefore we also hypothesized that this temperament trait might modify treatment outcome together with harmful alcohol use in depression [6].

\section{Methods}

\subsection{Participants}

In the period $2009-2013,242$ patients were screened for the study in the Finnish region of Southern Ostrobothnia (population 200,000). These patients were referred to psychiatric specialized care units (5 outpatients and 1 inpatient) due to depression, anxiety, self-destructiveness, insomnia or alcohol-related problems. To maximize clinical relevance, lenient inclusion criteria were used. Patients with at least moderate depressive symptomatology (Beck Depression Inventory [BDI] Version 1A, score $\geq 17$; [30]) were included in the study. Patients with organic brain disease or psychotic disorder (ICD-10 F2* diagnosis) were excluded. Their age range was 17-64 years (mean 38.8 years, SD \pm 12.2 ). A more detailed description of the sample is presented in Tables $1 \mathrm{a}$ and $1 \mathrm{~b}$, and of the study setting elsewhere (see ClinicalTrials.gov Identifier NCT02520271, Ostrobothnia Depression Study [ODS], 2016). The study was approved by the local Human Subjects Review Committee, and patients gave their informed written consent.

[Tables $1 \mathrm{a}$ and $1 \mathrm{~b}]$

\subsection{Procedures}

Sociodemographic data were collected and clinical assessments conducted at screening (the Alcohol Use Disorders Identification Test (AUDIT) [31] and the BDI). There was some dropout before the baseline assessment using the Mini International Neuropsychiatric Interview 5.0 (MINI; [32]) and the MontgomeryÅsberg Depression Rating Scale (MADRS) [33] and 228 (94\%) patients were assessed with MADRS at baseline. According to the MINI administered to 219 patients (data missing in 23 cases), $88.6 \%$ of the 
patients had MDD, 4.1\% dysthymic disorder, 5.5\% anxiety disorder and $0.4 \%$ alcohol use disorder (AUD) as their main diagnosis, and $1.4 \%$ of the patients did not meet any of the diagnostic criteria. Twelve percent $(12 \%)$ of the patients met the criteria for lifetime diagnosis of (hypo)manic episode. Sixty-three percent (63\%) of patients with mood disorder as their main diagnosis had comorbid anxiety disorders, corresponding well to comorbidity proportions found in other samples in Finnish psychiatric secondary services [34]. Six patients (3\%) with mood disorder as primary diagnosis had comorbid bulimia nervosa. Patients' categorical personality disorder diagnoses were not assessed. In the total sample, $33.6 \%$ of the patients reported that this was their first episode of MDD.

At baseline patients attended an appointment with a psychiatrist, where their medication was evaluated and changed if necessary. Antidepressant medication was prescribed to 206 patients (85\%) with mean fluoxetine equivalent daily doses of $33.0 \mathrm{mg}$ (SD \pm 18.3 ). Of these, $82 \%$ had either an SSRI or SNRI as a primary antidepressant. Adherence to antidepressants was monitored during the first six weeks of the study using a paper and pencil diary (for more information see [16]). All patients received behavioral activation therapy with trained clinical staff. The median number of therapy sessions with patients was 6 $(I Q R=3-11)$ with sessions taking place at 1 to 2 -week intervals. The treatment of patients with alcohol use problems (AUP, AUDIT scores $\geq 11$ ) was enhanced with motivational interviewing (median number of sessions 4, IQR = 3-6) at the start of the treatment according to a specific treatment intervention procedure (see ClinicalTrials.gov Identifier NCT02520271, Ostrobothnia Depression Study [ODS], 2016 and [35]). The cut-off point for AUDIT was chosen because in Finnish clinical practice it indicates a significantly increased risk of harm due to alcohol $[36,37]$. In the present study setting the aim was to identify individuals with marked alcohol use problems with high specificity to obtain motivational interview as an add-on psychosocial treatment.

After dropout at 6 weeks, 173 patients completed both the TCI-R and MADRS and were therefore eligible for inclusion in the main analysis of this study. Of these patients 61 (35\%) showed marked alcohol use problems (AUP, AUDIT scores $\geq 11$ ) roughly corresponding to the comorbidity ratio of 2:1 (of substance use 
disorders in depressed patients) observed in clinical samples where nearly one third of patients with major depressive disorder also have substance use disorders [38]. Of the 61 AUP patients MINI diagnosis had been assessed in 60 cases at baseline, 44 (73\%) of which had been diagnosed with lifetime AUD and of the other 112 patients (with AUDIT scores < 11) MINI diagnosis had been assessed in 107 cases, only $3(3 \%)$ of which had been diagnosed with lifetime AUD. Seven (16\%) patients that had been diagnosed with AUD had also other SUD, and two (2\%) patients without AUD were diagnosed with some other SUD. There was no exclusion of patients according to substance use. Alcohol use problems were more common in male patients: (males $69 \%$ vs. females $31 \%, \chi^{2}=31.5, p<0.001$; odds ratio $(O R)=6.6,95 \%$ confidence intervals $(\mathrm{Cl})=3.3-13.2)$. The follow-up included assessment of patients' MADRS scores again at 6-month and 24month time-points.

\subsection{Statistical methods}

Differences in continuous variables (AUDIT and MADRS scores, and TCI-R dimension scores) between dropouts and other patients were calculated with independent samples t-tests. Differences in nominal variables between dropouts and other patients and in the prevalence of AUP between genders were calculated with $\chi^{2}$ statistics.

This study was conducted according to the intention-to-treat protocol and in cases of dropout MADRS scores were imputed in the follow-up according to the last observation carried forward (LOCF) method. This replaced missing values in MADRS follow-up with their last observed values at earlier follow-up time points. This replaced missing MADRS values in 32 (17\%) cases at 6-month follow-up and in 93 (49\%) cases at 24month follow-up. Exploratory analysis between LOCF MADRS scores and temperament or character scores was performed with Pearson's correlation coefficients.

A linear mixed effects model was used for the repeated measurement testing in the main analysis of the study. This model predicted LOCF MADRS scores from 6 weeks to 6 months and to 24 months with the scores of the seven temperament and character dimensions (NS, HA, RD, P, SD, C, and ST; at six weeks) used as explaining variables and was adjusted with AUP, gender, and AUP $\mathrm{x}$ gender and AUP $\mathrm{x}$ time 
interactions. Individual-specific intercept and slope terms were used in the model. The -2 log likelihood information criteria was used in evaluating model fit and model with unstructured covariance structure was reported. Kenward-Roger adjustment of degrees of freedom was applied for estimates of fixed effects. The main analysis was performed with PROC MIXED, SAS version 9.4 (SAS Institute Inc., Cary, NC, USA) and all other analyses were performed with SPSS for Mac (version 24.0, IBM Inc., Armonk, New York, USA).

\section{Results}

The dropout rate in the study was $65(27 \%)$ at six weeks, $91(38 \%)$ at six months, and $147(61 \%)$ at 24 months, but no gender differences were seen at any assessment point. Of these patients, the clinical treatment had been concluded in co-operation with the patients in $10(11 \%)$ cases during the first 6 months and in 28 (19\%) cases before 24 months. The dropout analysis of the raw data revealed similar baseline MADRS scores in dropouts and other patients. Baseline AUDIT scores were higher in dropouts at both rating points ( 6 months: dropout $14.0 \pm 11.2$ vs. other patients $8.9 \pm 8.6, p<0.001 ; 24$ months: $12.0 \pm 10.5$ vs. $8.7 \pm 8.5, p=0.006$ for $t$-test). Dropouts also had lower Self-Directedness at baseline than did other patients in dropout analysis at 6 months: dropout $117.9 \pm 18.3$ vs. other patients $124.2 \pm 18.2, p=0.02$ for t-test, and had trending but non-significant difference at 24 months $(p=0.09)$. In the LOCF data there were no statistically significant differences between dropouts and other patients' 6-month LOCF MADRS scores in the 6-month dropout analysis ( $p=0.2$ for t-test). Dropouts had higher 24-month LOCF MADRS scores in the 24-month dropout analysis (dropout $12.9 \pm 8.9$ vs. other patients $8.3 \pm 7.6, p<0.001$ for t-test).

The main results emerging as predictors of depression treatment outcome (measured as MADRS scores) are presented in Table 2. Poorer outcome of depression was predicted by AUP x Time interaction together with low RD. The model resulted in steeper negative sloping of MADRS scores for non-AUP group when compared to AUP group $x$ Time. This means that alcohol use problems associated with poorer outcome of depression in the follow-up from 6 weeks to 6 months and to 24 months. Low RD was the only temperament or character trait that was associated with depression outcome as a predictor of poorer outcome in the follow-up from 6 weeks to 6 and 24 months. 
[Table 2]

The distributions (mean \pm SD) for LOCF MADRS scores were: 1) $13.6 \pm 8.5$ at 6 months and 2) $10.6 \pm 8.5$ at 24 months. The mean \pm SD for temperament and character dimensions at six weeks were: NS 99.7 $\pm 16.7 ; \mathrm{HA}$

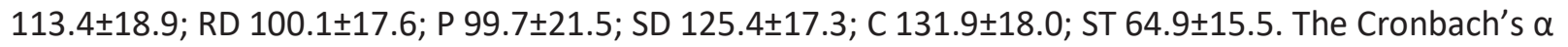
values for TCl-R dimensions have been reported elsewhere [16]. The MADRS scores, number of responders, remitted patients, and non-responders in follow-up are presented in Table 3.

[Table 3]

Pearson's correlation coefficients between RD, HA and SD and LOCF MADRS scores were statistically significant for RD and MADRS scores at 6 months $(r=-0.32, p<0.001)$, HA and MADRS scores at 6 months $(r$ $=0.18, p=0.02), \mathrm{SD}$ and MADRS scores at 6 months $(r=-0.27, p<0.001), \mathrm{HA}$ and MADRS scores at 24 months $(r=0.26, p=0.001)$, and SD and MADRS scores at 24 months $(r=-0.32, p<0.001)$.

\section{Discussion}

The main hypotheses in this study were that temperament and character traits together with harmful alcohol use (assessed in the early stages of treatment) explain outcome of depression over a period of two years. The main finding in this study was that poorer outcome of depression was predicted by low Reward Dependence. Alcohol use problems were also associated with poorer outcome of depression in the followup from 6 weeks to 6 months and to 24 months.

\subsection{Outcome of depression (from 6 weeks to 24 months)}

Poorer outcome of depression was predicted by low Reward Dependence in the linear mixed effects model adjusted with AUP, gender, AUP x gender and AUP $\mathrm{x}$ time. In contrast to some earlier studies our present and earlier findings suggest that Reward Dependence is associated with depression treatment outcome $[4,16,26]$. This difference in results could be explained by differences in patient samples, as patients with SUDs have been excluded in those earlier studies but not in this present one $[4,16,26]$. Our earlier and present results suggest that low RD predicts poorer outcome of depression particularly in depressed 
patients with comorbid alcohol use problems. In line with these findings, one study in which patients with prior SUD were not excluded resulted in MDD patients in remission having higher RD than currently depressed patients [24]. More specifically, the presence of SUD together with severe mental illness (SMI, including patients with MDD or schizophrenia) seems to be associated with low RD as dually diagnosed patients have lower RD than do SUD patients, but there is no significant difference in trait RD between patients with SMI or SUD without the comorbidity of the other [3]. The finding of an association between high RD (at 6 weeks) and better outcome of depression together with our earlier finding may be explained by depression state-dependent alterations in brain reward pathway functions in patients comorbid with SUD, and hence confirm a possible association between Reward Dependence and depression treatment outcome when alcohol use problems are taken into account $[16,39]$.

Although high HA and low SD have been associated earlier with risk of recurrences of depressive episodes as well as impaired depression treatment response $[4,5,25]$, surprisingly, in this study these temperament and character traits were not statistically significant in predicting the outcome of depression. One possible explanation for this would be that alcohol use problems in part mediate the association between these personality traits and depression outcome. This argument is justified because earlier findings have shown that AUDs are associated with both: 1) the development of more chronic courses of depressive symptomatology [1,2], and 2) high HA and low SD. More specifically high HA and low SD are both associated with dual diagnosis [3], and high HA also with more severe AUDs [22]. Moreover, it is plausible that lower ability to organized problem solving or greater distress associated with avoidant behavior that are associated with low SD and high HA, respectively, could predispose these individuals to a higher risk of alcohol use problems. Finding the Harm Avoidance and Self-Directedness statistically non-significant in predicting outcome of depression in our analysis with the control of AUP is in line with the argument that the association between high HA and low SD and poorer outcome of depression could be in part mediated by alcohol use problems. 
In the main analysis AUP $x$ Time was highly significant predictor of depression outcome and was associated with slower decline of MADRS scores in the follow-up when compared to non-AUP. This finding demonstrates the disruptive effect of alcohol use problems on the outcome of depression and is in line with earlier findings of the detrimental effect of AUDs on the course depression $[1,2]$. Novelty Seeking, Persistence, Self-Transcendence and Cooperativeness remained non-significant in predicting the outcome of depression when AUPs were taken into account. Although high NS could cause impaired response to antidepressive treatment because it is associated with more severe SUD symptomatology $[6,18,20]$, it seems that high NS is not an important trait in the association with poorer outcome of depression when AUP are controlled. Similarly low Persistence seems to be a marker for more severe or chronic SUD $[21,23,40]$, but it was not a significant marker in predicting depression outcome in this study. The results from different patient samples have been partially contradictory regarding associations between trait ST and depressive symptomatology and the present results suggest that ST alone is not significant in predicting depression outcome nor together with AUP $[4,15,26,27]$. Although high Cooperativeness could be protective of depression and associated with better drinking outcomes in depressed alcohol-dependent patients $[4,6]$ it was a non-significant predictor of the outcome of depression together with alcohol use problems in this study and this association should be verified in larger samples. As gender has not been associated with severity or recurrence of MDD episodes [2], it was expected that it would likewise not predict depression outcome in this sample of more diverse depressed patients.

\subsection{Strengths and limitations}

In the mixed effects multivariate model we analyzed the factors predicting the outcome of depression (MADRS scores) across three different time-points (at 6 weeks, 6 months and 24 months). Because depressive episodes are on average of 6 months' duration, the follow-up period up to 6 months is a good marker for responsiveness to anti-depressive treatment, whereas follow-up period up to 24 months is likely to reflect considerably more the long-term fluctuation in symptoms, also including relapses and recurrences of depressive episodes [41,42]. It was important to study the outcome across both of these 
outcomes, because in the majority of cases recovery from depression is not a straightforward process but instead takes a fluctuating course with remissions, relapses, and recurrences of episodes [42]. Moreover, it is possible that traits associated with responsiveness to treatment could in part differ from the traits predisposing to recurrence of episodes and this could be interesting hypothesis to test in future studies in naturalistic samples [4].

As there was marked dropout in this study, especially at 24-month follow-up, data imputation was conducted for the main analysis. The method of last observation carried forward was used even though it could result in more conservative results. It is possible that the LOCF method used in this study did not detect all relapses, but the risk that it would have markedly affected the results in this study was considered low because the naturalistic study setting allowed patients to continue in the study even if they had failed to attend at some earlier point.

According to the theory of pathoplasticity, personality has a causal effect on the clinical course and outcome of depression; e.g., individual personality determines how patients recover from depression [7]. However, the association between depression and temperament is more complex, and the depressive state also leads to higher HA [5]. In addition, in this patient sample RD also showed potential state-dependent alterations [16], and increase in SD seems to be associated with recovery from depression [26]. Because depressive symptoms have been shown to alter temperament and character profiles, the six-week TCI-R assessments were considered as better representing the patients' long-term temperament and character profiles and were used in the GLM analyses as explanatory variables.

Due to the naturalistic setting we had more lenient inclusion criteria in this study than in other studies with depressed patients, and because of that our patient sample likely included more patients with different subtypes of depressive symptomatology [16]. The patients in this study also had marked comorbidities including life-time diagnosis of (hypo)mania in some cases, and a large proportion had AUD. Moreover, the seasonal patterns of depressive symptomatology or personality disorders were not controlled for in the main analysis. Therefore, these results are not directly applicable to "pure" MDD patients but are more 
easily generalizable to the mixed populations seen in psychiatric secondary services. A few patients had other SUDs (in addition to AUDs) that were not specified but could have had an effect on the personality profile of these patients. However, because of the low number of patients with other SUDs this have not likely affected the results markedly. According to the treatment procedure only a portion of the patients underwent motivational interviewing in addition to behavioral activation therapy, and, although temperament is considered to reflect relatively stable traits, it is possible that this difference in treatment affected the results. Dropout in follow-up was linked with high AUDIT and low SD at baseline, which may also have affected the results.

\subsection{Conclusions}

Possibly due to the modifying effect of alcohol use problems, high Reward Dependence was associated with better anti-depressive treatment outcome at 6 months. Harm Avoidance and Self-Directedness did not predict depression outcome when alcohol use problems were controlled.

\section{Acknowledgments}

This study was supported by a grant from Finland's Psychiatric Research Association and the Finnish Psychiatric Association (Dr. Paavonen) and funds from the Southern Ostrobothnia Hospital District (EVO1114) and the State of Finland. The authors express their warmest thanks to research nurses Susanna Ahola, Marja Koivumäki, and Kati Huhtala for their skillful efforts and to all participants for their collaboration during the study. 


\section{References}

[1] Holzel L, Harter M, Reese C, Kriston L. Risk factors for chronic depression--a systematic review. J Affect Disord 2011;129:1-13.

[2] Burcusa SL, lacono WG. Risk for recurrence in depression. Clin Psychol Rev 2007;27:959-985.

[3] Fernandez-Mondragon S, Adan A. Personality in male patients with substance use disorder and/or severe mental illness. Psychiatry Res 2015;228:488-494.

[4] Farmer RF, Seeley JR. Temperament and character predictors of depressed mood over a 4-year interval. Depression \& Anxiety 2009;26:371-381.

[5] Kampman O, Poutanen O. Can onset and recovery in depression be predicted by temperament? A systematic review and meta-analysis. J Affect Disord 2011;135:20-27.

[6] Foulds JA, Mulder RT, Newton-Howes G, Adamson SJ, Boden JM, Sellman JD. Personality Predictors of Drinking Outcomes in Depressed Alcohol-Dependent Patients. Alcohol Alcohol 2016;51:296-301.

[7] Klein DN, Kotov R, Bufferd SJ. Personality and depression: explanatory models and review of the evidence. Annu rev clin psychol 2011;7:269-295.

[8] Cloninger CR, Svrakic DM, Przybeck TR. A Psychobiological Model of Temperament and Character. Arch Gen Psychiatry 1993;50:975-990.

[9] Cloninger CR. Biology of personality dimensions. Current Opinion in Psychiatry 2000;13:611-616.

[10] Cloninger CR. A unified biosocial theory of personality and its role in the development of anxiety states. Psychiatr Dev 1986;4:167-226.

[11] Farmer A, Mahmood A, Redman K, Harris T, Sadler S, McGuffin P. A sib-pair study of the Temperament and Character Inventory scales in major depression. Arch Gen Psychiatry 2003;60:490-496.

[12] Naito M, Kijima N, Kitamura T. Temperament and Character Inventory ( $\mathrm{TCl}$ ) as predictors of depression among Japanese college students. J Clin Psychol 2000;56:1579-1585.

[13] Peirson AR, Heuchert JW. The relationship between personality and mood: Comparison of the BDI and the TCI. Personality and Individual Differences 2001;30:391-399.

[14] Joffe RT, Bagby RM, Levitt AJ, Regan JJ, Parker JD. The Tridimensional Personality Questionnaire in major depression. Am J Psychiatry 1993;150:959-960.

[15] Spittlehouse JK, Pearson JF, Luty SE, Mulder RT, Carter JD, McKenzie JM, et al. Measures of temperament and character are differentially impacted on by depression severity. J Affect Disord 2010;126:140-146.

[16] Paavonen V, Luoto K, Koivukangas A, Lassila A, Leinonen E, Kampman O. Temperament and character profiles associated with depression and treatment response in patients with or without comorbid substance abuse. Psychiatry Res 2016;245:250-258. 
[17] Martinotti G, Cloninger CR, Janiri L. Temperament and character inventory dimensions and anhedonia in detoxified substance-dependent subjects. The American Journal of Drug and Alcohol Abuse 2008;34:177183.

[18] Evren C, Durkaya M, Evren B, Dalbudak E, Cetin R. Relationship of relapse with impulsivity, novelty seeking and craving in male alcohol-dependent inpatients. Drug and Alcohol Review 2012;31:81-90.

[19] Zilberman ML, Tavares H, el-Guebaly N. Relationship between craving and personality in treatmentseeking women with substance-related disorders. BMC Psychiatry 2003;3.

[20] Howard MO, Kivlahan D, Walker RD. Cloninger's tridimensional theory of personality and psychopathology: applications to substance use disorders. J Stud Alcohol 1997;58:48-66.

[21] Evren C, Evren B, Dalbudak E. Alexithymia and personality dimensions in relation to depression and anxiety in male alcohol-dependent inpatients. International Journal of Psychiatry in Clinical Practice 2009;13:3-10.

[22] Sher KJ, Bartholow BD, Wood MD. Personality and Substance Use Disorders: A Prospective Study. Journal of Consulting \& Clinical Psychology 2000;68:818-829.

[23] Rae AM, Joyce PR, Luty SE, Mulder RT. The effect of a history of alcohol dependence in adult major depression. Journal of Affective Disorders 2002;70:281-290.

[24] Nery FG, Hatch JP, Nicoletti MA, Monkul SE, Najt P, Matsuo KM, et al. Temperament and character traits in major depressive disorder: Influence of mood state and recurrence of episodes. Depress Anxiety 2009;26:382-388.

[25] Asano T, Baba H, Kawano R, Takei H, Maeshima H, Takahashi $Y$, et al. Temperament and character as predictors of recurrence in remitted patients with major depression: A 4-year prospective follow-up study. Psychiatry Res 2015;225:322-325.

[26] Corruble E, Duret C, Pelissolo A, Falissard B, Guelfi JD. Early and delayed personality changes associated with depression recovery? A one-year follow-up study. Psychiatry Res 2002;109:17-25.

[27] Richter J, Polak T, Eisenmann M. Depressive mood and personality in terms of temperament and character among the normal population and depressive inpatients. Personality and Individual Differences 2003;35:917-927.

[28] Yoon S, Kim T, Kim D. Personality dimensions measured by the Temperament and Character Inventory in male subjects with substance-related disorders. Clinical Psychopharmacology and Neuroscience 2007;5:70-75.

[29] Ball SA, Tennen H, Poling JC, Kranzler HR, Rounsaville BJ. Personality, temperament, and character dimensions and the DSM-IV personality disorders in substance abusers. Journal of Abnormal Psychology 1997; 106:545-553.

[30] Beck AT, Steer RA, Ball R, Ranieri W. Comparison of Beck Depression Inventories -IA and -II in psychiatric outpatients. J Pers Assess 1996;67:588-597.

[31] Bohn MJ, Babor TF, Kranzler HR. The Alcohol Use Disorders Identification Test (AUDIT): validation of a screening instrument for use in medical settings. J Stud Alcohol 1995;56:423-432. 
[32] Sheehan DV, Lecrubier Y, Sheehan KH, Amorim P, Janavs J, Weiller E, et al. The Mini-International Neuropsychiatric Interview (M.I.N.I.): the development and validation of a structured diagnostic psychiatric interview for DSM-IV and ICD-10. J Clin Psychiatry 1998;59:22-33;quz.

[33] Montgomery SA, Asberg M. A new depression scale designed to be sensitive to change. Br J Psychiatry 1979;134:382-389.

[34] Melartin TK, Rytsala HJ, Leskela US, Lestela-Mielonen PS, Sokero TP, Isometsa ET. Current comorbidity of psychiatric disorders among DSM-IV major depressive disorder patients in psychiatric care in the Vantaa Depression Study. J Clin Psychiatry 2002;63:126-134.

[35] Lindholm LH, Koivukangas A, Lassila A, Kampman O. Early assessment of implementing evidence-based brief therapy interventions among secondary service psychiatric therapists. Eval Program Plann 2015;52:182-188.

[36] Luoto KE, Koivukangas A, Lassila A, Kampman O. Outcome of patients with dual diagnosis in secondary psychiatric care. Nord J Psychiatry 2016;70:470-476.

[37] Babor TF, Higgins-Biddle JC, Saunders JB, Monteiro MG. Alcohol Use Disorders Identification Test, The: Guidelines for Use in Primary Health Care. 2nd ed. Geneva: Department of Mental Health and Substance Dependence, World Health Organization; 2001.

[38] Davis LL, Frazier E, Husain MM, Warden D, Trivedi M, Fava M, et al. Substance use disorder comorbidity in major depressive disorder: a confirmatory analysis of the STAR*D cohort. Am J Addict 2006;15:278-285.

[39] Koob GF, Le Moal M. Addiction and the brain antireward system. Annu Rev Psychol 2008;59:29-53.

[40] Sellman JD, Mulder RT, Sullivan PF, Joyce PR. Low persistence predicts relapse in alcohol dependence following treatment. J Stud Alcohol 1997;58:257-263.

[41] Kupfer DJ. Long-term treatment of depression. J Clin Psychiatry 1991;52:28-34.

[42] Solomon DA, Keller MB, Leon AC, Mueller TI, Shea MT, Warshaw M, et al. Recovery from major depression. A 10-year prospective follow-up across multiple episodes. Arch Gen Psychiatry 1997;54:10011006.

Ostrobothnia Depression Study (ODS). A Naturalistic Follow-up Study on Depression and Related Substance Use Disorders. https://clinicaltrials.gov/ct2/show/NCT02520271, (accessed: April 04, 2017) 
Table 1a. Sociodemographic data on the patient sample

\begin{tabular}{lcccccc}
\hline & \multicolumn{2}{c}{ Men } & \multicolumn{2}{c}{ Women } & \multicolumn{3}{c}{ Total } \\
& $N$ & $\%$ & $N$ & $\%$ & $N$ & $\%$ \\
\hline Total & 94 & 38.8 & 148 & 61.2 & 242 & 100 \\
\hline Marital status & & & & & & \\
Single & 34 & 36.9 & 39 & 29.1 & 73 & 32.3 \\
Married or cohabiting & 39 & 42.4 & 71 & 53.0 & 110 & 48.7 \\
Divorced & 19 & 20.7 & 21 & 15.7 & 40 & 17.7 \\
Widowed & 0 & 0 & 3 & 2.2 & 3 & 1.3 \\
\hline Education & & & & & & \\
Primary school & 4 & 4.3 & 3 & 2.2 & 7 & 3.1 \\
Comprehensive school & 27 & 29.3 & 28 & 20.7 & 55 & 24.2 \\
Tertiary education & 10 & 10.9 & 25 & 18.5 & 35 & 15.4 \\
Vocational school & 33 & 35.9 & 54 & 40.0 & 87 & 38.3 \\
Upper secondary education & 10 & 10.9 & 7 & 5.2 & 17 & 7.5 \\
Polytechnic or university & 8 & 8.7 & 18 & 13.3 & 26 & 11.5 \\
\hline Work status before sick leave & & & & & & \\
Employed & 39 & 42.9 & 67 & 50.0 & 106 & 47.1 \\
Unemployed & 41 & 45.1 & 30 & 22.4 & 71 & 31.6 \\
Housewife/husband & 0 & 0 & 10 & 7.5 & 10 & 4.4 \\
Pensioner & 5 & 5.5 & 11 & 8.2 & 16 & 7.1 \\
Student & 6 & 6.6 & 16 & 11.9 & 22 & 9.8 \\
\hline Self-reported history of & 62 & 67.4 & 90 & 65.7 & 152 & 66.4 \\
depression episode & & & & & & \\
\hline First degree family history of & 33 & 35.9 & 56 & 41.5 & 89 & 39.2 \\
depression & & & & & & \\
\hline First degree family history of & 4 & 4.3 & 10 & 7.4 & 14 & 6.2 \\
bipolar disorder & & & & & & \\
\hline
\end{tabular}

Table 1b. Baseline scores on BDI and AUDIT by gender

\begin{tabular}{cccc}
\hline & Men & Women & Total \\
\hline $\begin{array}{c}\text { Baseline BDI mean } \\
( \pm S D)\end{array}$ & $28.5(6.80)$ & $27.5(7.59)$ & $27.9(7.30)$ \\
$\begin{array}{c}\text { Baseline AUDIT mean } \\
( \pm \text { SD })\end{array}$ & $15.1(10.59)$ & $7.9(8.30)$ & $10.7(9.88)$ \\
\hline
\end{tabular}

Abbreviations: BDI = Beck Depression Inventory; AUDIT = Alcohol Use Disorder Identification Test 
Table 2. Predictors of depression outcome (LOCF MADRS scores across the follow-up from 6 weeks to 6 months and to 24 months) ${ }^{\mathrm{a}}$

\begin{tabular}{lcccc} 
Fixed effects & \multicolumn{4}{c}{ LOCF MADRS scores } \\
\cline { 2 - 5 } Intercept & Estimate $^{\mathrm{b}}$ & $\mathrm{SE}$ & $t$ & $p$ \\
NS at 6 weeks & $\mathbf{2 0 . 0 7}$ & $\mathbf{1 0 . 0 4}$ & $\mathbf{2 . 0 0}$ & $\mathbf{0 . 0 4 9}$ \\
HA at 6 weeks & 0.04 & 0.03 & 1.16 & 0.25 \\
RD at 6 weeks & 0.06 & 0.04 & 1.70 & 0.09 \\
P at 6 weeks & $-\mathbf{0 . 1 1}$ & $\mathbf{0 . 0 4}$ & $\mathbf{- 3 . 0 3}$ & $\mathbf{0 . 0 0 3}$ \\
SD at 6 weeks & -0.03 & 0.03 & -1.10 & 0.27 \\
C at 6 weeks & -0.06 & 0.04 & -1.59 & 0.11 \\
ST at 6 weeks & 0.02 & 0.04 & 0.56 & 0.58 \\
\hline Male gender & 0.05 & 0.03 & 1.49 & 0.14 \\
AUP & 0.24 & 1.76 & 0.14 & 0.23 \\
AUP x Male gender & 3.67 & 1.64 & 2.25 & 0.12 \\
AUP x Time & -3.24 & 2.19 & -1.48 & 0.14 \\
non-AUP x Time & $-\mathbf{0 . 1 0}$ & $\mathbf{0 . 0 5}$ & $\mathbf{- 1 . 9 8}$ & $\mathbf{0 . 0 0 0 2}$ \\
\hline
\end{tabular}

$-2 \times \log$-likelihood $=3530$ for the SAS input code and output results

${ }^{a}$ Results from the linear mixed effects model with temperament and character dimension scores, gender, AUP, and AUP x Gender and AUP x Time interactions as explanatory variables

${ }^{\mathrm{b}} B$ for the temperament and character variables effect on the dependent variable

Abbreviations: $L O C F=$ Last observation carried forward; NS = Novelty Seeking; HA = Harm Avoidance; RD = Reward Dependence; $\mathrm{P}=$ Persistence; $\mathrm{SD}=$ Self-Directedness; $\mathrm{C}=$ Cooperativeness; $\mathrm{ST}=$ Self-

Transcendence; MADRS = Montgomery-Åsberg Depression Rating Scale; AUP = alcohol use problems

Significant results are presented in bold face. 
Table 3. MADRS scores in raw data, LOCF data, and by patient subgroup and number of responders, patients in remission and non-responders at baseline and follow-up

\begin{tabular}{|c|c|c|c|c|}
\hline & baseline & 6 weeks & 6 months & 24 months \\
\hline Raw data MADRS scores (mean $\pm S D$ ) & $\begin{array}{l}23.2 \pm 6.7 \\
n=228\end{array}$ & $\begin{array}{l}16.9 \pm 8.0 ; \\
n=188\end{array}$ & $\begin{array}{l}13.1 \pm 8.7 \\
n=156\end{array}$ & $\begin{array}{l}8.3 \pm 7.6 \\
n=95\end{array}$ \\
\hline Response* $(n, \%)$ & & & $69,44 \%$ & $64,67 \%$ \\
\hline Remission** $(\mathrm{n}, \%)$ & & & $49,31 \%$ & $50,53 \%$ \\
\hline LOCF data MADRS scores, $\mathrm{n}=188$ & & & $13.6 \pm 8.5$ & $10.6 \pm 8.5$ \\
\hline Response* $(n, \%)$ & & & $80,43 \%$ & $112,60 \%$ \\
\hline Remission** (n, \%) & & & $52,28 \%$ & $80,43 \%$ \\
\hline
\end{tabular}

MADRS scores of patient subgroups (Raw data scores):

\begin{tabular}{|c|c|c|c|c|}
\hline non-AUP (mean $\pm S D$ ) & $\begin{array}{l}22.9 \pm 6.7 \\
(n=136)\end{array}$ & $\begin{array}{l}16.7 \pm 8.0 \\
(n=123)\end{array}$ & $\begin{array}{l}12.8 \pm 8.7 \\
(n=105)\end{array}$ & $\begin{array}{l}6.7 \pm 6.2 \\
(n=66)\end{array}$ \\
\hline AUP $($ mean $\pm S D)$ & $\begin{array}{l}23.7 \pm 6.7 \\
(n=92)\end{array}$ & $\begin{array}{l}17.2 \pm 8.1 \\
(n=65)\end{array}$ & $\begin{array}{l}13.7 \pm 8.7 \\
(n=51)\end{array}$ & $\begin{array}{l}12.0 \pm 9.2 \\
(n=29)\end{array}$ \\
\hline Female (mean $\pm S D$ ) & $\begin{array}{l}22.3 \pm 6.8 \\
(n=137)\end{array}$ & $\begin{array}{l}15.7 \pm 7.8 \\
(n=112)\end{array}$ & $\begin{array}{l}11.6 \pm 8.2 \\
(n=97)\end{array}$ & $\begin{array}{l}7.1 \pm 6.6 \\
(n=64)\end{array}$ \\
\hline Male (mean $\pm S D$ ) & $\begin{array}{l}24.6 \pm 6.3 \\
(n=91)\end{array}$ & $\begin{array}{l}18.6 \pm 8.0 \\
(\mathrm{n}=76)\end{array}$ & $\begin{array}{l}15.6 \pm 9.1 \\
(n=59)\end{array}$ & $\begin{array}{l}10.8 \pm 8.9 \\
(n=31)\end{array}$ \\
\hline proportion of men $(n, \%)$ & $91,40 \%$ & $76,40 \%$ & $59,38 \%$ & $31,33 \%$ \\
\hline proportion of AUP patients ( $n, \%)$ & $92,40 \%$ & $65,35 \%$ & $51,33 \%$ & $29,31 \%$ \\
\hline $\begin{array}{l}\text { Number of patients with increase in } \\
\text { symptoms from baseline }(n, \%)\end{array}$ & & & $23,15 \%$ & $3,3 \%$ \\
\hline
\end{tabular}

Abbreviations: AUP= Alcohol use problems; MADRS = Montgomery-Åsberg Depression Rating Scale; LOCF = last observation carried forward

*at least 50\% MADRS score decline from baseline; **MADRS scores $<8$ 